\title{
ZMIZ1 promotes the proliferation and migration of melanocytes in vitiligo
}

\author{
MENG LI ${ }^{1 *}$, YIBIN FAN $^{2 *}$, YUTONG WANG ${ }^{1}$, JINHUA XU $^{3}$ and HUI XU ${ }^{1}$ \\ ${ }^{1}$ Department of Dermatology, Shanghai Ninth People's Hospital, Shanghai Jiaotong University, School of Medicine, \\ Shanghai 200011; ${ }^{2}$ Department of Dermatology, Zhejiang Provincial People's Hospital, People's Hospital of \\ Hangzhou Medical College, Hangzhou, Zhejiang 310014; ${ }^{3}$ Department of Dermatology, \\ Huashan Hospital, Fudan University, Shanghai 200041, P.R. China
}

Received September 25, 2019; Accepted March 25, 2020

DOI: $10.3892 / \mathrm{etm} .2020 .8849$

\begin{abstract}
Genome wide association studies have revealed that the zinc finger MIZ-type containing 1 (ZMIZ1) is involved in the pathogenesis of vitiligo; however, the underlying mechanism remains unclear. The present study aimed to investigate the effects of ZMIZ1 on the proliferation, apoptosis and migration of the human melanocyte cell lines PIG1 and PIG3V. ZMIZ1 overexpression and knockdown PIG1 and PIG3V cell models were established by lentivirus infection, and the effects of ZMIZ1 on cell proliferation and apoptosis were determined using an MTT assay and flow cytometry, respectively. Furthermore, the expression levels of proliferation- and apoptosis-associated proteins were analyzed using western blotting. Additionally, Transwell assays were performed to determine the effect of ZMIZ1 on the migration of PIG1 and PIG3V cells. Finally, the effect of ZMIZ1 on cytoskeletal remodeling in PIG1 and PIG3V cells was analyzed using immunocytochemistry. The overexpression of ZMIZ1 promoted the proliferation and inhibited the apoptosis of PIG1 and PIG3V cells, whereas the genetic knockdown of ZMIZ1 resulted in the opposite effects. Furthermore, ZMIZ1 overexpression increased the migration, whereas the knockdown of ZMIZ1 inhibited the migration and altered remodeling of the actin cytoskeleton in PIG1 and PIG3V cells. In conclusion, the results of the present study suggest that ZMIZ1 regulates
\end{abstract}

Correspondence to: Dr Jinhua Xu, Department of Dermatology, Huashan Hospital, Fudan University, 12 Urumqi Middle Road, Shanghai 200041, P.R. China

E-mail: xjhhsyy@163.com

Dr Hui Xu, Department of Dermatology, Shanghai Ninth People's Hospital, Shanghai Jiaotong University, School of Medicine, 639 Zhizaoju Road, Shanghai 200011, P.R. China

E-mail: xusunan@hotmail.com

*Contributed equally

Key words: zinc finger MIZ-type containing 1, vitiligo, melanocytes, proliferation, migration the proliferation, apoptosis and migration of PIG1 and PIG3V cells, and indicate that ZMIZ1 may serve as a potential therapeutic target for vitiligo.

\section{Introduction}

Vitiligo is a common skin disorder characterized by melanocyte dysfunction in the epidermis of the skin, which results in the appearance of white spots on the skin of affected patients (1). The prevalence of vitiligo is $\sim 1 \%$ in the USA (2) and $\sim 0.56 \%$ in China (3). Although vitiligo is not life-threatening, it is associated with a significant psychological burden for patients (4) and it may result in large financial costs for public health systems. In fact, it is estimated that the cost of vitiligo is $\sim 175$ million dollars per year in the USA (5). The pathogenesis of vitiligo remains unclear and currently, there is no gold standard treatment for the disease (6). Therefore, there is an urgent requirement for the identification of novel therapeutic targets and the development of new methods to treat vitiligo with an improved therapeutic efficacy.

Previously, clinical and epidemiological investigations have demonstrated that vitiligo is a complex disease that can be affected by various environmental and genetic factors, such as heredity and gene mutation (7-9). In addition, it was reported that NLR family pyrin domain containing 1 (NLRP1) and the histocompatibility complex are associated with an increased risk of developing vitiligo (10-12). Notably, genome-wide analysis previously suggested that zinc finger MIZ-type containing 1 (ZMIZ1) is significantly associated with the occurrence and development of vitiligo (13). ZMIZ1, also termed hZiMP10, is located in the $10 \mathrm{q} 22.3$ region of the chromosome (14). ZMIZ1 belongs to the protein inhibitor of activated STAT (PIAS) family and it encodes a PIAS-like protein containing 1,067 amino acid residues (15). Similar to other PIAS proteins, ZMIZ1 contains a ring finger region termed Miz, which serves an important role in protein-protein interactions (16). However, the exact role of ZMIZ1 in vitiligo remains unknown and requires further investigation.

Therefore, the present study aimed to investigate the role of ZMZ1 in vitiligo, in addition to the related mechanism. ZMIZ1 overexpression and knockdown melanocyte cell lines were established, and the effects of ZMIZ1 on the prolifera- 
tion, apoptosis, migration and invasion of melanocytes were investigated. The results of the present study may provide a theoretical basis for the clinical application of ZMIZ1 as a potential therapeutic target for the treatment of vitiligo.

\section{Materials and methods}

Cell culture. The human normal melanocyte cell line PIG1 and human vitiligo melanocyte cell line PIG3V were purchased from ScienCell Research Laboratories, Inc. Cells were cultured in Medium 254 (Gibco; Thermo Fisher Scientific, Inc.), supplemented with $0.5 \% \mathrm{FBS}$, and $100 \mathrm{U} / \mathrm{ml}$ penicillin and $100 \mathrm{U} / \mathrm{ml}$ streptomycin Cells were maintained in a humidified incubator at $37^{\circ} \mathrm{C}$ and $5 \% \mathrm{CO}_{2}$. The medium was changed every other day.

Lentiviral infection. The gene fragment of ZMIZ1 and three short hairpin RNA (shRNA/sh) interference fragments, sh-ZMIZ1-1, sh-ZMIZ1-2 and sh-ZMIZ1-3 (purchased from Shanghai GenePharma Co., Ltd.), targeting ZMIZ1 were subcloned into lentiviral vectors (pGLVH1/GFP+Puro; Shanghai GenePharma Co., Ltd.), alongside the overexpression negative control (OE NC; empty vector) and the sh-NC. Packaging plasmid (pAX2), envelope plasmid (pMD2.G) and pGLVH1/GFP+Puro, 293T cells (seeded onto the cell culture plates with $5 \times 10^{6}$ cells/well) (ScienCell Research Laboratories, Inc.) were infected with these lentiviral vectors as previously described (17). The sequences of the shRNAs were as follows: Sh-ZMIZ1-1, 5'-GGAGAGCCCAACTATGGAAAC-3'; sh-ZMIZ1-2, 5'-GCCCATCAAGTCGGACTTAC-3'; sh-ZMIZ1-3, 5'-GCCAGATGATCATGCCCAATG-3', sh-NC, 5'-TAATGCTATCCGTCTAATC-3'. 293T cells (Cell Bank of the Chinese Academy of Sciences; $3 \times 10^{6}$ cells $/ 10 \mathrm{~cm}$ dish) were transfected using Lipofectamine ${ }^{\mathrm{TM}} 3000$ (Invitrogen; Thermo Fisher Scientific, Inc.) according to the manufacturer's protocol. The following ratio was used for vector transfection: Lentiviral vectors: packaging plasmid: envelope plasmid; $10 \mu \mathrm{g}$ : $7.5 \mu \mathrm{g}: 3 \mu \mathrm{g}$ for $72 \mathrm{~h}$ at $37^{\circ} \mathrm{C}$ in an incubator before the 293T lentiviral supernatant was harvested by centrifugation at $200 \mathrm{x}$ g for $5 \mathrm{~min}$ at room temperature. PIG1 and PIG3V cells $\left(2 \times 10^{5}\right.$ cells $\left./ \mathrm{ml}\right)$ in six-well plates were infected with the $293 \mathrm{~T}$ lentiviral supernatant in the presence of $5 \mu \mathrm{g} / \mathrm{ml}$ polybrene (Beyotime Institute of Biotechnology). Cells were collected $48 \mathrm{~h}$ after infection for further analysis, and the infection efficiency was determined by fluorescence microscopy (magnification, $\mathrm{x} 400$ ).

Cells were divided into 7 groups: i) Control (Con) group (uninfected cells); ii) OE NC group; iii) OE ZMIZ1 group; iv) sh-NC group; v) sh-ZMIZ1-1 group; vi) sh-ZMIZ1-2 group; and vii) sh-ZMIZ1-3 group.

$R N A$ extraction and reverse transcription-quantitative PCR $(R T-q P C R)$. To determine the mRNA expression levels of ZMIZ1 in PIG1 and PIG3V cells, total RNA was extracted from the cells using the RNeasy Mini kit (Qiagen GmbH), according to the manufacturer's protocol. Total RNA was reverse transcribed into cDNA using the PrimeScript RT Master mix (Takara Biotechnology Co., Ltd.) according to the manufacturer's protocol. The expression levels of ZMIZ1 were quantified by qPCR using the SYBR-Greem Premix Ex Taq kit
(Takara Biotechnology, Co., Ltd.) according to the manufacturer's protocol and an ABI 7500 Biosystems thermocycler. The following thermocycling conditions were used: Initial denaturation at $94^{\circ} \mathrm{C}$ for $5 \mathrm{~min}, 36$ cycles at $94^{\circ} \mathrm{C}$ for $20 \mathrm{sec}, 54^{\circ} \mathrm{C}$ for $20 \mathrm{sec}$ and $72^{\circ} \mathrm{C}$ for $20 \mathrm{sec}$. ZMIZ1 mRNA levels were quantified using the $2^{-\triangle \Delta \mathrm{Cq}}$ method (18) and normalized to the internal reference gene GAPDH. The following primers sequences were used: ZMIZ1 forward, 5'-TGTTTGACGGTGGTCAGTCG-3' and reverse, 5'-CTTGTCTCGGTTTGCAGCAC-3'; and GAPDH forward, 5'-TGTGGGCATCAATGGATTTGG-3' and reverse, 5'-ACACCATGTATTCCGGGTCAAT-3'.

Western blotting. Total protein was extracted from PIG1 and PIG3V cells using RIPA lysis buffer (Beyotime Institute of Biotechnolohy). Total protein was quantified using a bicinchoninic acid assay kit (Beyotime Institute of Biotechnology) and $20 \mu \mathrm{g}$ protein/lane was separated by $8 \%$ SDS-PAGE. The separated proteins were subsequently transferred onto PVDF membranes and blocked with 5\% non-fat dry milk in Trisbuffered saline (TBS; pH 7.4) containing 0.05\% Tween-20 at room temperature for $1 \mathrm{~h}$. The membranes were incubated with the following primary antibodies overnight at $4^{\circ} \mathrm{C}$ : antiZMIZ1 (1:1,000; cat. no. abs126964a; Absin, Biotechnology Co., Ltd.), anti-Bcl-2 (1:1,000; cat. no. ab196495; Abcam), anticaspase-3 (1:1,000; cat. no. 9662; Cell Signaling Technology, Inc.), anti-cleaved caspase-3 antibody (1:1,000; cat. no. 9661; Cell Signaling Technology, Inc.) anti-GAPDH (1:2,000; cat. no. 60004-1-1; ProteinTech Group, Inc.) and anti- $\alpha$-tubulin (1:2,000; cat. no. 66031-1-1g; ProteinTech Group, Inc.). Following the primary antibody incubation, the membranes were incubated with horseradish peroxidase-conjugated secondary antibodies (anti-rabbit antibody; 1:2,000; cat. no. A0208 and anti-mouse antibody; 1:2,000; cat. no. A0216; both from Beyotime Institute of Biotechnology) at room temperature for $1 \mathrm{~h}$ and then washed using TBS-Tween-20. Protein bands were visualized using a SuperSignal West Pico Chemiluminescent substrate (Pierce; Thermo Fisher Scientific, Inc.) and the chemiluminescence signals were detected using a Tanon-5200 Imaging system (Tanon Science and Technology, Co., Ltd.). $\alpha$-tubulin or GAPDH served as the loading control. Densitometric analysis was performed using ImageJ (v1.46; National Institutes of Health).

Cell proliferation assay. The MTT assay was performed $48 \mathrm{~h}$ post-lentiviral infection to determine the effect of ZMIZ1 on the proliferation of PIG1 and PIG3V cells. Briefly, cells were harvested by centrifugation $(300 \mathrm{x} \mathrm{g})$ at $4^{\circ} \mathrm{C}$ for $5 \mathrm{~min}$, resuspended, seeded into 96 -well plates $\left(5 \times 10^{3}\right.$ cells/well). Next, $5 \mathrm{mg} / \mathrm{ml}$ MTT solution was added into each well and the cells were incubated for an additional $4 \mathrm{~h}$ in a humidified incubator at $37^{\circ} \mathrm{C}$ and $5 \% \mathrm{CO}_{2}$. Finally, the absorbance at a wavelength of $490 \mathrm{~nm}$ was measured using a microplate reader.

Flow cytometric analysis of apoptosis. The effect of ZMIZ1 on the apoptosis of PIG1 and PIG3V cells was determined using the Annexin $\mathrm{V}$ apoptosis detection kit (Nanjing KeyGen Biotech Co., Ltd.), according to the manufacturer's protocol. Briefly, cells $\left(5 \times 10^{5}\right.$ cells $\left./ \mathrm{ml}\right)$ were harvested and double-stained with 7-aminoactinomycin D and Annexin V/ allophycocyanin at room temperature for $15 \mathrm{~min}$. Apoptotic 
A
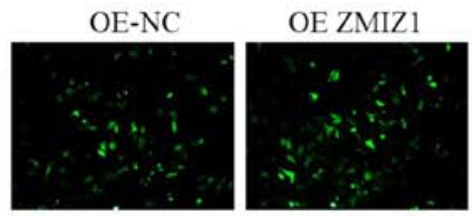

PIG1
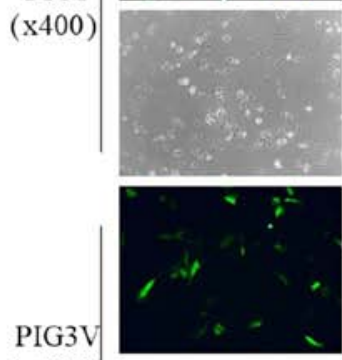

$(\mathrm{x} 400)$
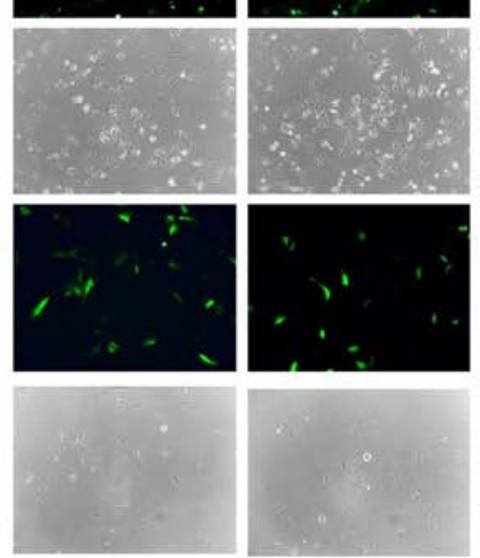

PIGl cells

B

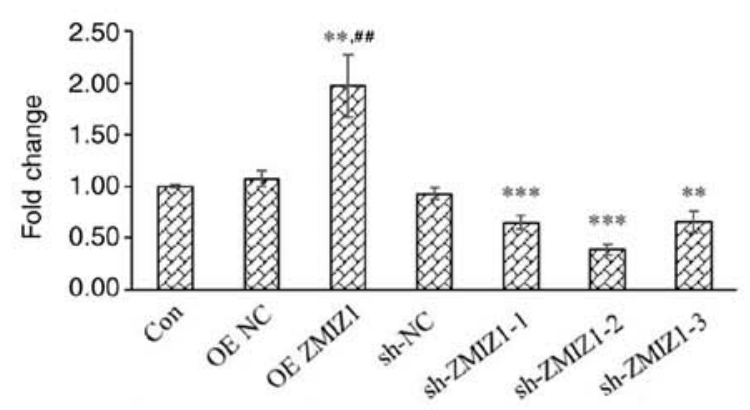

C

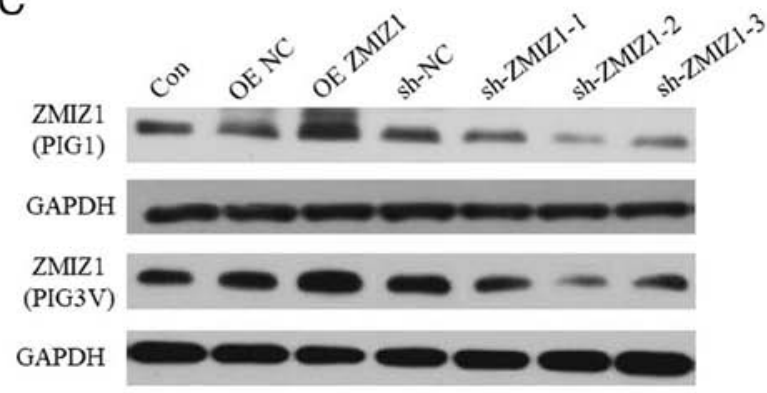

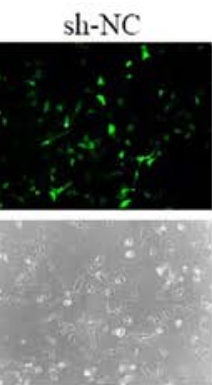
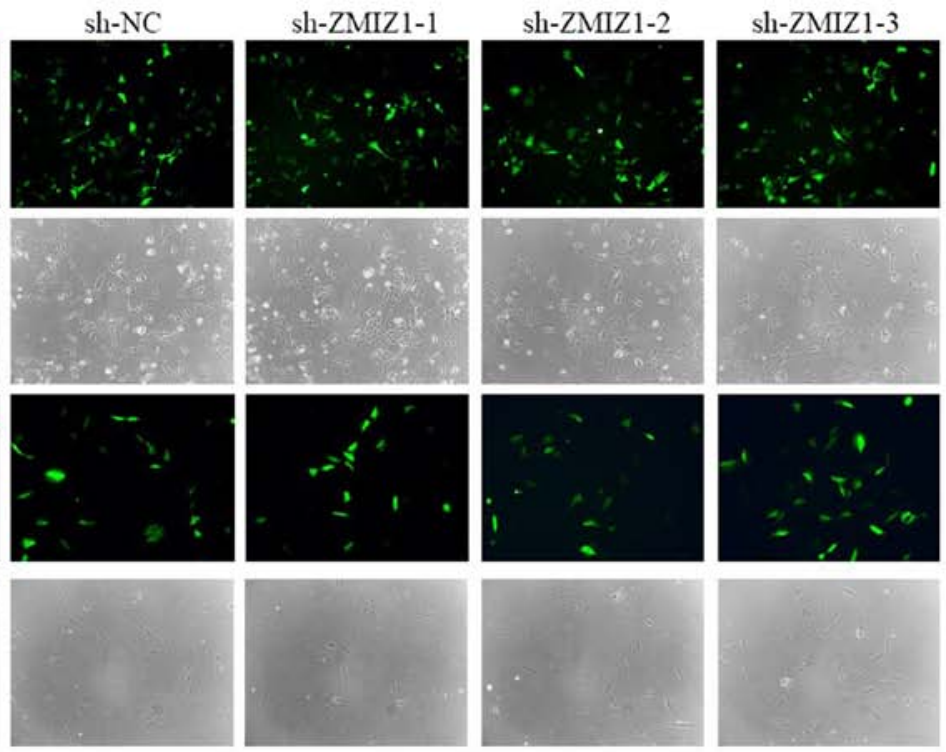

PIG3V cells
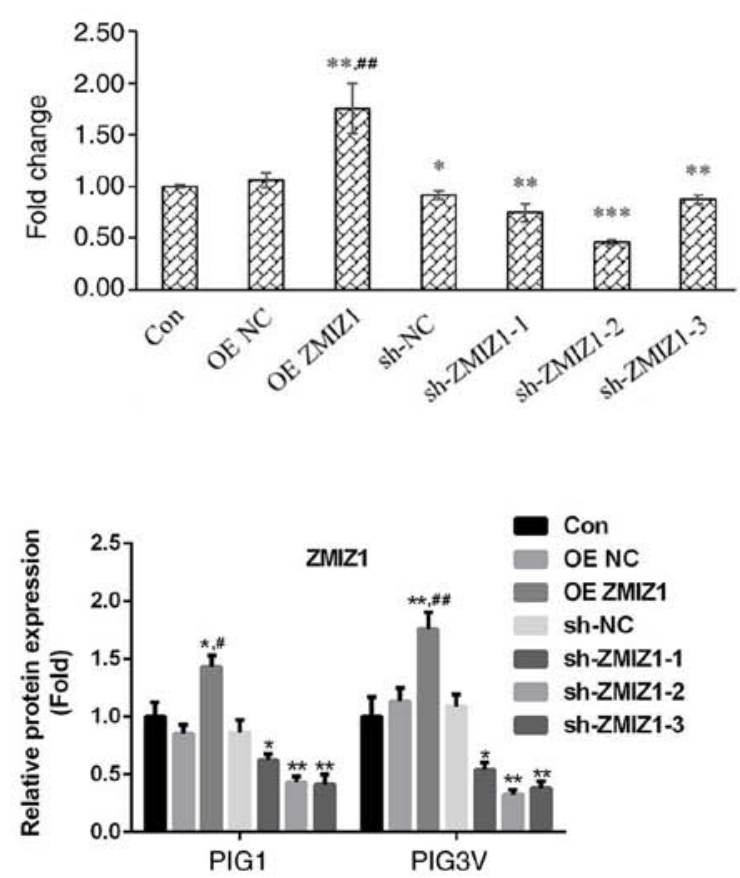

Figure 1. Establishment of the ZMIZ1 knockdown and overexpression cell models. (A) Representative fluorescent images of the lentivirus-infected cells (magnification, x400). (B) mRNA expression levels of ZMIZ1 were analyzed in the different groups using reverse transcription-quantitative PCR. (C) Protein expression levels of ZMIZ1 were analyzed in the different groups using western blotting. ${ }^{*} \mathrm{P}<0.05,{ }^{* *} \mathrm{P}<0.01$ and ${ }^{* * *} \mathrm{P}<0.001$ vs. Con. ${ }^{\#} \mathrm{P}<0.05$ and ${ }^{\# \#} \mathrm{P}<0.01$ vs. OE NC. Con, control; ZMIZ1, zinc finger MIZ-type containing 1; OE, overexpression; NC, negative control; sh, short hairpin RNA.

cells were subsequently analyzed in the different groups using a BD FACS Calibur flow cytometer (BD Biosciences) and FlowJo software (v10.4; FlowJo LLC).

Transwell assay. The effect of ZMIZ1 on the migration of PIG1 and PIG3V cells was determined using a Transwell assay. PIG1 and PIG3V cells were harvested and plated into the upper chambers of 24-well Transwell plates (Corning, Inc.) at a density of $5 \times 10^{4}$ cells/well serum-free Medium 254. The lower chamber was filled with Medium 254 containing 10\% FBS. Following the incubation at $37^{\circ} \mathrm{C}$ for $24 \mathrm{~h}$, the migratory cells in the lower chamber were fixed in $4 \%$ paraformaldehyde for
$20 \mathrm{~min}$ at room temperature and stained with crystal violet for $10 \mathrm{~min}$ at room temperature. Stained cells were viewed under a light microscope (magnification, x200). The number of migratory cells was counted and quantified using ImageJ (v1.46; National Institutes of Health).

Immunocytochemistry. PIG1 and PIG3V cells $\left(3 \times 10^{4}\right.$ cells/ coverslip) were seeded on glass coverslips and washed with PBS, fixed in $4 \%$ paraformaldehyde for $30 \mathrm{~min}$ at room temperature and permeabilized with $0.3 \%$ Triton $\mathrm{X}-100$ for $20 \mathrm{~min}$ at room temperature. Subsequently, the cells were blocked with 1\% BSA (Gibco; Thermo Fisher Scientific, 
A

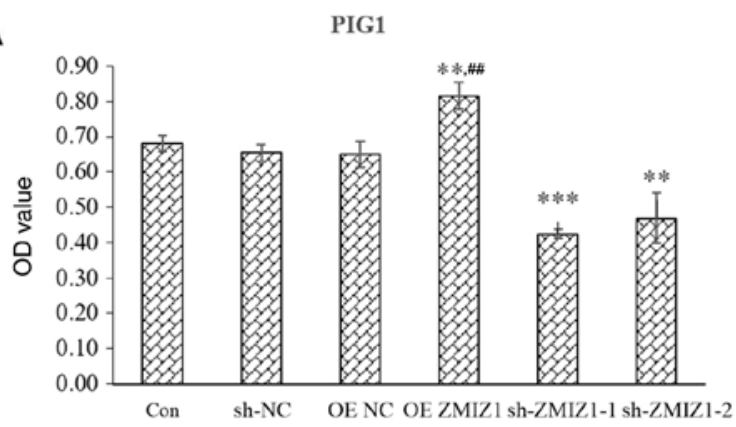

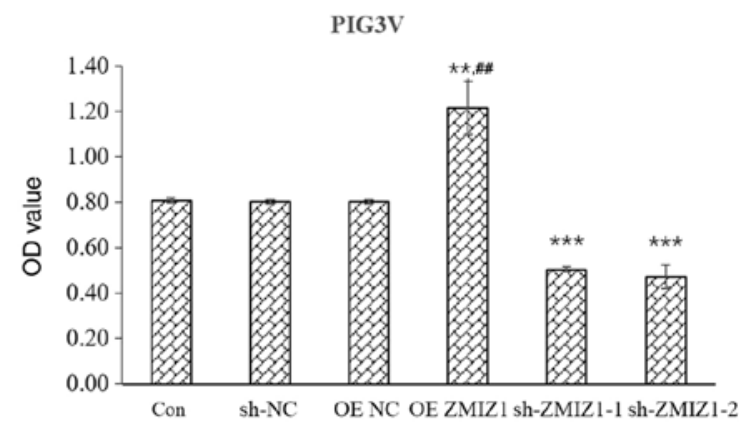

B
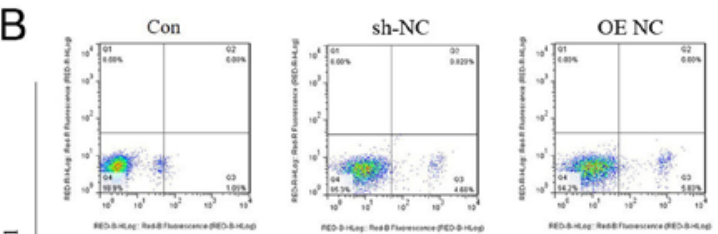

$\bar{\Xi}$
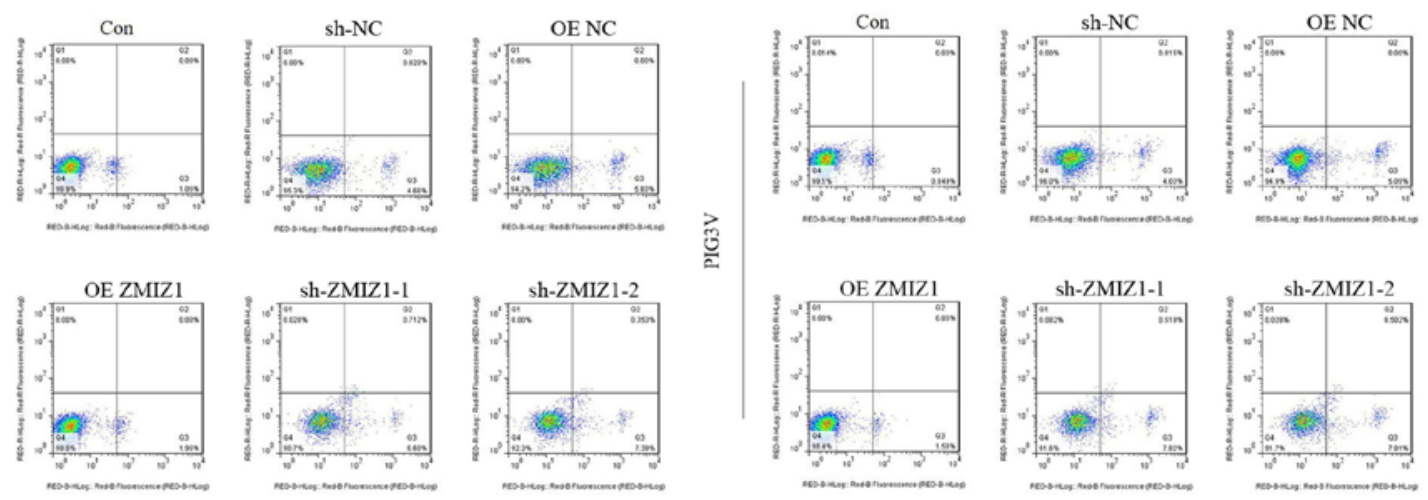

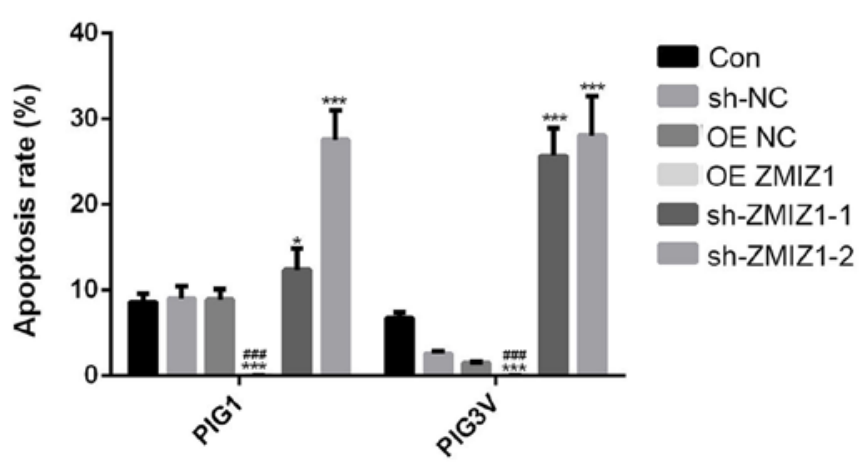

Figure 2. Effects of ZMIZ1 on the proliferation and apoptosis of PIG1 and PIG3V cells. (A) Effect of ZMIZ1 on the proliferation of PIG1 and PIG3V cells was analyzed using an MTT assay. (B) Effect of ZMIZ1 on the apoptosis of PIG1 and PIG3V cells was analyzed using flow cytometry. ${ }^{*} \mathrm{P}<0.05$, ${ }^{* * *} \mathrm{P}<0.01$ and ${ }^{* * * *} \mathrm{P}<0.001$ vs. Con. ${ }^{\# \#} \mathrm{P}<0.01$ and ${ }^{\# \# \#} \mathrm{P}<0.001$ vs. OE NC. Con, control; sh, short hairpin RNA; OE, overexpression; NC, negative control; ZMIZ1, zinc finger MIZ-type containing 1; OD, optical density.

Inc.) for $30 \mathrm{~min}$ at room temperature. The Phalloidin-iFluor 488 reagent (cat. no. ab176753; Abcam) was used to stain the cells, according to the manufacturer's protocol, for $30 \mathrm{~min}$ at room temperature. Stained cells were imaged under a confocal microscope (magnification, x63).

Statistical analysis. Statistical analysis was performed using SPSS software (v22.0; IBM Corp.) and data are presented as the mean \pm SD of $\geq 3$ independent experimental repeats. Statistical differences among the groups were determined using one-way ANOVA followed by Tukey's post hoc test. $\mathrm{P}<0.05$ was considered to indicate a statistically significant difference.

\section{Results}

Establishment of ZMIZ1 knockdown and overexpression in vitro cell models. PIG1 and PIG3V cells were infected with lentiviral vectors to establish ZMIZ1 knockdown and overexpression cell models. Fluorescence was observed in each infected group, suggesting that the lentivirus infection had been successful (Fig. 1A). Moreover, RT-qPCR and western blotting analysis were performed to evaluate the efficiency of the infection. ZMIZ1 expression levels were significantly increased in the OE ZMIZ1 group at both the mRNA Fig. 1B) and protein (Fig. 1C) levels. Conversely, sh-ZMIZ1-1, sh-ZMIZ1-2 and sh-ZMIZ1-3 significantly decreased the mRNA and protein expression levels of ZMIZ1 in both PIG1 and PIG3V cells compared with the sh-NC (Fig. 1B and C). Based on these results, sh-ZMIZ1-1 and sh-ZMIZ1-2 were used for ZMIZ1 knockdown in subsequent experiments.

Effects of ZMIZI on the proliferation and apoptosis of PIGI and PIG3V cells in vitro. The effect of ZMIZ1 on cell proliferation was analyzed using a MTT assay and flow cytometry $48 \mathrm{~h}$ post-lentiviral infection. The overexpression of ZMIZ1 

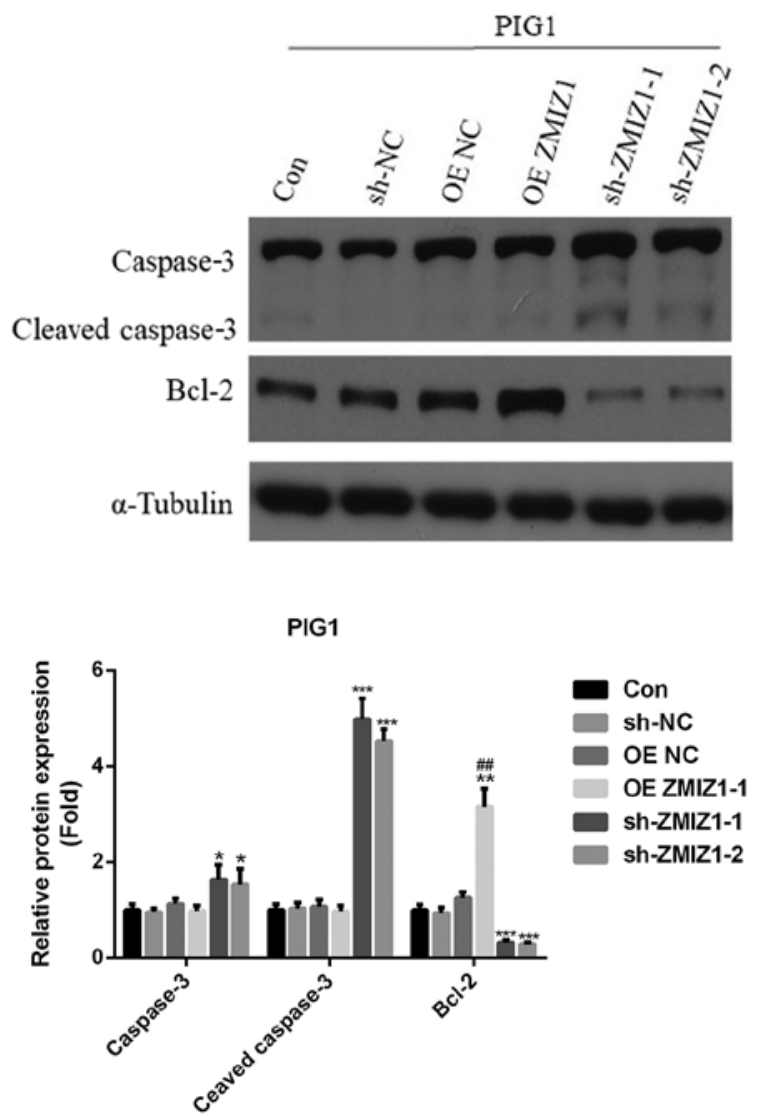
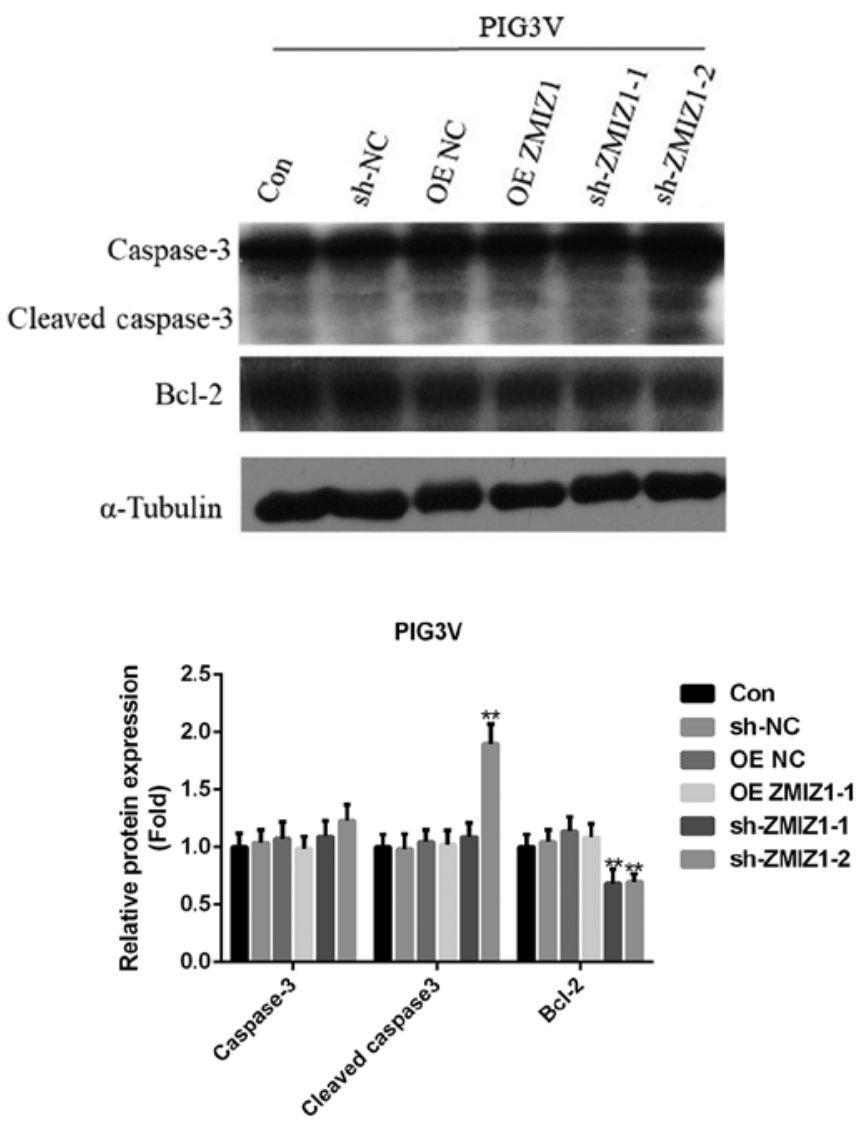

Figure 3. ZMIZ1 regulates the expression levels of Bcl-2 and caspase-3 in PIG1 and PIG3V cells. Effect of ZMIZ1 on the expression levels of proliferation- and apoptosis-related proteins in PIG1 and PIG3V cells was analyzed using western blotting. ${ }^{*} \mathrm{P}<0.05,{ }^{* *} \mathrm{P}<0.01$ and ${ }^{* * * *} \mathrm{P}<0.001$ vs. Con. ${ }^{\# \#} \mathrm{P}<0.01$ vs. OE NC. Con, control; sh, short hairpin RNA; OE, overexpression; NC, negative control; ZMIZ1, zinc finger MIZ-type containing 1; OD, optical density.

significantly increased the proliferation $(\mathrm{P}<0.01$; Fig. $2 \mathrm{~A})$ and decreased the apoptotic rate (Fig. 2B) compared with the Con group. Conversely, the genetic knockdown of ZMIZ1 significantly inhibited the proliferation (Fig. 2A) and induced the apoptosis of PIG1 and PIG3V cells in vivo (Fig. 2B) compared with the Con group.

ZMIZ1 regulates the expression levels of Bcl-2 and caspase- 3 in PIG1 and PIG3V cells in vitro. The effects of ZMIZ1 on the expression levels of proliferation- and apoptosis-associated proteins were analyzed using western blotting. The overexpression of ZMIZ1 significantly increased the expression levels of the anti-apoptotic protein Bcl-2 in PIG1 cells compared with the Con group (Fig. 3). Conversely, the genetic knockdown of ZMIZ1 significantly decreased the expression levels of Bcl-2, and increased the expression levels of the proapoptotic protein caspase- 3 and its activated form, cleaved caspase-3, in PIG1 cells (Fig. 3). Moreover, the genetic knockdown of ZMIZ1 significantly decreased the expression levels of Bcl-2 in PIG3V cells compared with the Con group and sh-ZMIZ1-2 significantly increased the expression levels of cleaved caspase- 3 in PIG3V cells compared with the Con group. On the other hand, the overexpression of ZMIZ1 had no significant effects on the expression levels of caspase-3 and cleaved caspase-3 in PIG1 and PIG3V cells, and the genetic knockdown of ZMIZ1 had no significant effects on the expression levels of caspase- 3 in PIG3V cells (Fig. 3).
Effect of ZMIZ1 overexpression and knockdown on the migration of PIG1 and PIG3V melanocytes. The effects of ZMIZ1 on the migration of PIG1 and PIG3V cells were investigated using a Transwell assay. The overexpression of ZMIZ1 significantly increased the migratory ability of PIG1 and PIG3V cells compared with the Con group (Fig. 4), whereas the genetic knockdown of ZMIZ1 demonstrated the opposite effects (Fig. 4).

ZMIZ1 affects the remodeling of actin cytoskeleton in PIG1 and PIG3V melanocytes in vitro. The remodeling of the actin cytoskeleton has been considered as a necessary process for the migration of different cell types, including melanocytes $(19,20)$. Therefore, the effects of ZMIZ1 on actin remodeling in the cytoskeleton were investigated using immunocytochemistry. Genetic knockdown of ZMIZ1 resulted in morphology of the cells to become irregular, and the cell shape tend to be more rounded, indicating the degradation of the cytoskeleton and apoptosis of the cell (Fig. 5).

\section{Discussion}

The present study successfully established zinc finger MIZ-type containing 1 (ZMIZ1) overexpression and knockdown PIG1 and PIG3V model cell lines, which were subsequently used to investigate the effects of ZMIZ1 on the biological behaviors of melanocytes. ZMIZ1 promoted proliferation and migration 

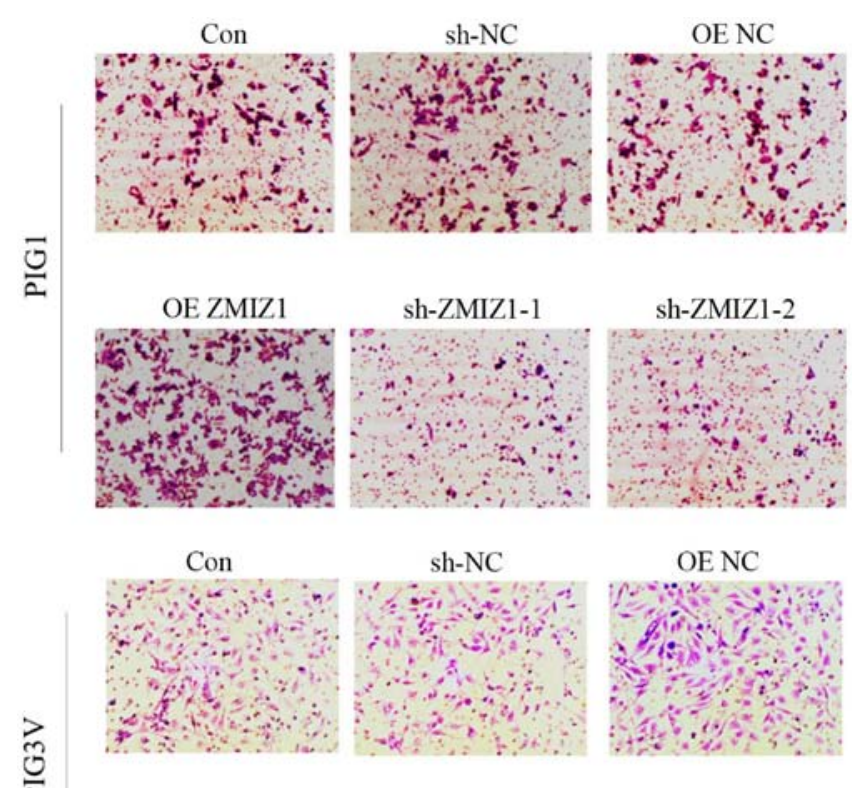

sh-NC

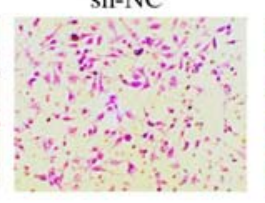

OE ZMIZ1

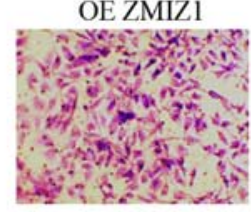

sh-ZMIZ1-1

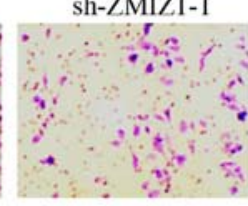

sh-ZMIZ1-2

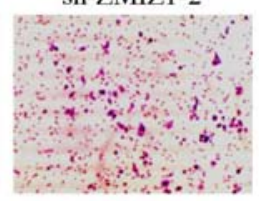

OE NC

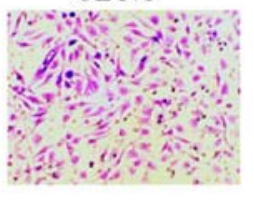

sh-ZMIZ1-2
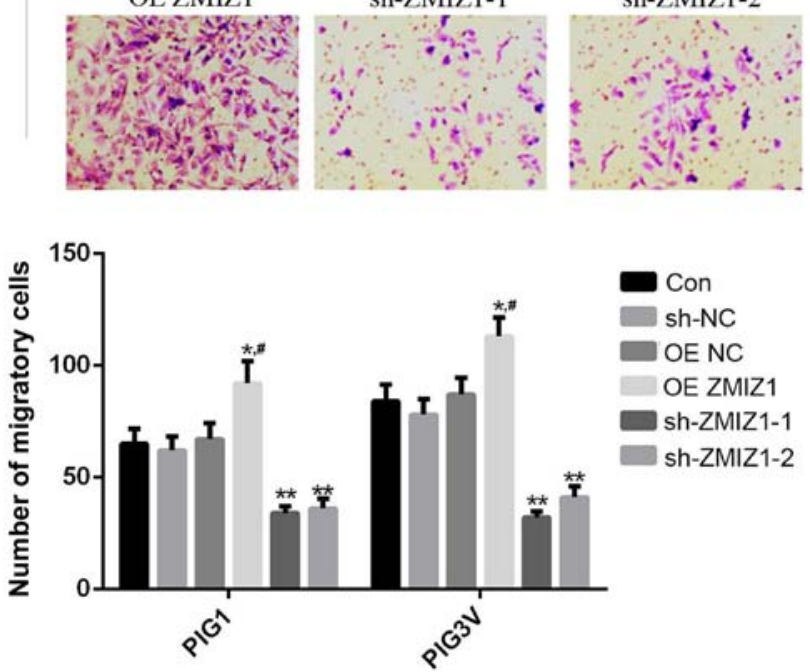

Figure 4. ZMIZ1 regulates the migration of PIG1 and PIG3V melanocytes. Effect of ZMIZ1 on the migration of PIG1 and PIG3V cells was analyzed using a Transwell assay. Magnification, $x 200 .{ }^{~} \mathrm{P}<0.05$ and ${ }^{* *} \mathrm{P}<0.01$ vs. Con. ${ }^{*} \mathrm{P}<0.05$ vs. OE NC. Con, control; sh, short hairpin RNA; OE, overexpression; NC, negative control; ZMIZ1, zinc finger MIZ-type containing 1; OD, optical density.

and inhibited apoptosis of PIG1 and PIG3V cells, suggesting that ZMIZ1 may be a potential therapeutic target for the treatment of vitiligo. A previous report discovered that PIAS3, a member of the PIAS family, inhibited the transcriptional activity of melanocyte inducing transcription factor and STAT3 in vivo (21), and it has been further reported that both of these transcription factors may serve important roles in the growth and maintenance of melanocytes (21). Similar to other PIAS family proteins, ZMIZ1 has been found to enhance the transcriptional activity of the SMAD3/4 complex; this subsequently activated the TGF- $\beta /$ SMAD signaling pathway, which serves an important role in the regulation of melanocyte proliferation, differentiation and regulation $(22,23)$. Moreover, it was also suggested that ZMIZ1 may induce autoreactive T cells and the loss of tolerance to melanocyte antigens during vitiligo (24). The aforementioned studies indicated that ZMIZ1, as well as other PIAS members, may regulate the

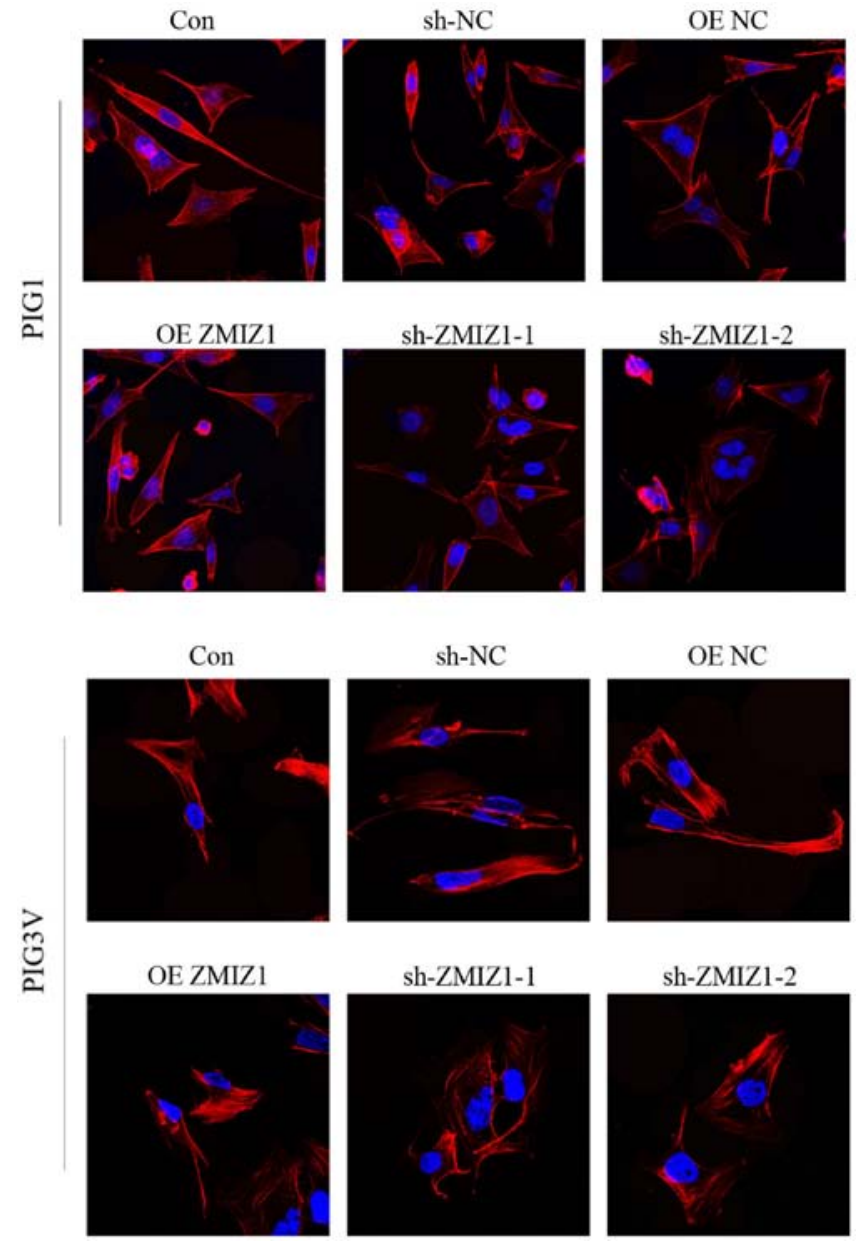

Figure 5. Effect of ZMIZ1 on the expression levels of F-actin cytoskeleton protein in PIG1 and PIG3V cells in vitro was analyzed using immunocytochemistry staining. Magnification, x63. Con, control; sh, short hairpin RNA; $\mathrm{OE}$, overexpression; NC, negative control; ZMIZ1, zinc finger MIZ-type containing 1; OD, optical density.

growth and function of melanocytes, and that the aberrant expression levels of ZMIZ1 may cause melanocyte dysfunction, which may lead to the development of vitiligo. However, reports on the effects of ZMIZ1 on melanocytes are limited.

Increasing evidence has suggested that the impaired proliferation and increased apoptosis of melanocytes may lead to the occurrence and progression of vitiligo $(25,26)$. In the present study, the effect of ZMIZ1 on the proliferation and apoptosis of the human melanocyte cell lines PIG1 and PIG3V cells was investigated. It was discovered that the overexpression of ZMIZ1 significantly increased the proliferation and significantly decreased the apoptotic rate of the cells, whereas the genetic knockdown of ZMIZ1 exhibited the opposite effects. Bcl-2 is a well-known anti-apoptotic protein and caspase-3 is considered as a proapoptotic protein $(27,28)$. The results obtained in the present study discovered that the overexpression of ZMIZ1 increased the expression levels of Bcl-2, while the genetic knockdown of ZMIZ1 decreased the expression levels of Bcl-2, in addition to increasing the expression levels of caspase-3 and its activated form cleaved caspase-3 in PIG1 and PIG3V cells. Taken together, these results suggested that ZMIZ1 may affect the proliferation and apoptosis of melanocytes in vivo. 
Human melanocytes originate from the neural crest and following proliferation, migrate to the nearby epidermis or the hair matrices to produce melanin (29). Therefore, migration is an important step in the function of melanocytes. In the case of vitiligo, functional melanocytes are destroyed in skin lesions and consequently, promoting the migration of functional melanocytes to the depigmented area is the main aim of current anti-vitiligo strategies $(26,30)$. In the present study, the overexpression of ZMIZ1 increased the migratory ability and the remodeling of the actin cytoskeleton in PIG1 and PIG3V cells, while the genetic knockdown of ZMIZ1 resulted in the opposite effects. These results indicated that ZMIZ1 may facilitate the migration and cytoskeleton rearrangement of melanocytes, suggesting that the overexpression of ZMIZ1 may be a potential strategy to recruit functional melanocytes to the site of the vitiliginous skin.

Nevertheless, the present study only performed in vivo cellular studies, therefore the role of ZMIZ1 in the pathogenesis of vitiligo requires further investigation using in vivo animal studies and clinical analysis in the future.

In conclusion, to the best of our knowledge, the present study was the first to suggest that ZMIZ1 may regulate the proliferation, apoptosis and migration of human melanocytes in vitro. The results obtained in the present study provided novel evidence to indicate that ZMIZ1 may serve as a novel therapeutic target in vitiligo.

\section{Acknowledgements}

Not applicable.

\section{Funding}

The present study was supported by funds from the Initial Funding for Outstanding Young People in Zhejiang Provincial People's Hospital (grant no. ZRY2018C004) and the Shanghai Natural Science Foundation (grant no. 16ZR1419200).

\section{Availability of data and materials}

The datasets used and/or analyzed during the present study are available from the corresponding author on reasonable request.

\section{Authors' contributions}

ML performed the experiments and wrote the manuscript; YF and YW performed the experiments; JX and HX designed the study and revised the manuscript. All authors read and approved the final manuscript.

\section{Ethics approval and consent to participate}

Not applicable.

\section{Patient consent for publication}

Not applicable.

\section{Competing interests}

The authors declare that they have no competing interests.

\section{References}

1. Yadav AK, Singh P and Khunger N: Clinicopathologic analysis of stable and unstable vitiligo: A study of 66 cases. Am J Dermatopathol 38: 608-613, 2016.

2. Shi H, Lin B, Huang Y, Wu J, Zhang H, Lin C, Wang Z, Zhu J, Zhao Y, Fu X, et al: Basic fibroblast growth factor promotes melanocyte migration via activating PI3K/Akt-Rac1-FAK-JNK and ERK signaling pathways. IUBMB Life 68: 735-747, 2016.

3. Wang X, Du J, Wang T, Zhou C, Shen Y, Ding X, Tian S, Liu Y, Peng G, Xue S, et al: Prevalence and clinical profile of vitiligo in China: A community-based study in six cities. Acta Derm Venereol 93: 62-65, 2013.

4. Grimes PE and Miller MM: Vitiligo: Patient stories, selfesteem, and the psychological burden of disease. Int J Womens Dermatol 4: 32-37, 2018.

5. Hadi A, Wang JF, Uppal P, Penn LA and Elbuluk N: Comorbid diseases of vitiligo: A 10-year cross-sectional retrospective study of an urban US population. J Am Acad Dermatol 82: 628-633, 2020.

6. Yuan X, Meng D, Cao P, Sun L, Pang Y, Li Y, Wang X, Luo Z, Zhang L and Liu G: Identification of pathogenic genes and transcription factors in vitiligo. Dermatol Ther (Heidelb) 32: e13025, 2019.

7. Galeone M, Colucci R, Dragoni F and Moretti S: Can environmental factors contribute in triggering vitiligo and associated autoimmune thyroid diseases? Clinical series possibly related to the Chernobyl nuclear accident. G Ital Dermatol Venereol 153: 729-730, 2018

8. Laddha NC, Dwivedi M, Gani AR, Mansuri MS and Begum R: Tumor necrosis factor B (TNFB) genetic variants and its increased expression are associated with vitiligo susceptibility. PLoS One 8: e81736, 2013.

9. Al-Harthi F, Zouman A, Arfin M, Tariq M and Al-Asmari A: Tumor necrosis factor- $\alpha$ and $-\beta$ genetic polymorphisms as a risk factor in Saudi patients with vitiligo. Genet Mol Res 12: 21962204, 2013.

10. Veiga-CastelliL, Oliveira ML,Pereira A,DebortoliG, Marcorin L, Fracasso N, Silva G, Souza A, Massaro J, Simões AL, et al: HLA-G polymorphisms are associated with non-segmental vitiligo among Brazilians. Biomolecules 9: 9, 2019.

11. Yi X, Cui T, Li S, Yang Y, Chen J, Guo S, Jian Z, Li C, Gao T, Liu L, et al: Identification of the risk HLA-A alleles and autoantigen in Han Chinese vitiligo patients and the association of CD8+T cell reactivity with disease characteristics. Med Sci Monit 24: 6489-6497, 2018.

12. Li J, Yan M, Zhang Y, Feng C, Wang H, Wang C and Sun L: Metaanalysis of the association between NLRP1 polymorphisms and the susceptibility to vitiligo and associated autoimmune diseases. Oncotarget 8: 88179-88188,2017.

13. Sun Y, Zuo X, Zheng X, Zhou F, Liang B, Liu H, Chang R, Gao J, Sheng Y, Cui H, et al: A comprehensive association analysis confirms ZMIZ1 to be a susceptibility gene for vitiligo in Chinese population. J Med Genet 51: 345-353, 2014.

14. Yang SK, Hong M, Choi H, Zhao W, Jung Y, Haritunians T, Ye BD, Kim KJ, Park SH, Lee I, et al: Immunochip analysis identification of 6 additional susceptibility loci for Crohn's disease in Koreans. Inflamm Bowel Dis 21: 1-7, 2015.

15. Rakowski LA, Garagiola DD, Li CM, Decker M, Caruso S, Jones M, Kuick R, Cierpicki T, Maillard I and Chiang MY: Convergence of the ZMIZ1 and NOTCH1 pathways at C-MYC in acute T lymphoblastic leukemias. Cancer Res 73: 930-941, 2013.

16. Peng Y, Lee J, Zhu C and Sun Z: A novel role for protein inhibitor of activated STAT (PIAS) proteins in modulating the activity of Zimp7, a novel PIAS-like protein, in androgen receptor-mediated transcription. J Biol Chem 285: 11465-11475, 2010.

17. Xu L, Zhang Z, Xie T, Zhang $X$ and Dai T: Inhibition of BDNF-AS Provides Neuroprotection for Retinal Ganglion Cells against Ischemic Injury. PLoS One 11: e0164941, 2016.

18. Livak KJ and Schmittgen TD: Analysis of relative gene expression data using real-time quantitative PCR and the 2(-Delta Delta C(T)) Method. Methods 25: 402-408, 2001.

19. Schnoor M: Endothelial actin-binding proteins and actin dynamics in leukocyte transendothelial migration. J Immunol 194: 3535-3541, 2015.

20. Wang DG, Xu XH, Ma HJ, Li CR, Yue XZ, Gao J and Zhu WY: Stem cell factor combined with matrix proteins regulates the attachment and migration of melanocyte precursors of human hair follicles in vitro. Biol Pharm Bull 36: 1317-1325, 2013. 
21. Thingnes J, Lavelle TJ, Gjuvsland AB, Omholt SW and Hovig E: Towards a quantitative understanding of the MITFPIAS3-STAT3 connection. BMC Syst Biol 6: 11, 2012.

22. Kim HJ, Choi CP, Uhm YK, Kim YI, Lee JW, Yoon SH, Chung JH and Lee MH: The association between endothelin-1 gene polymorphisms and susceptibility to vitiligo in a Korean population. Exp Dermatol 16: 561-566, 2007.

23. Jeong KH, Shin MK, Uhm YK, Kim HJ, Chung JH and Lee MH: Association of TXNDC5 gene polymorphisms and susceptibility to nonsegmental vitiligo in the Korean population. Br J Dermatol 162: 759-764, 2010

24. Ben Ahmed M, Zaraa I, Rekik R, Elbeldi-Ferchiou A, Kourda N Belhadj Hmida N, Abdeladhim M, Karoui O, Ben Osman A Mokni M, et al: Functional defects of peripheral regulatory $\mathrm{T}$ lymphocytes in patients with progressive vitiligo. Pigment Cell Melanoma Res 25: 99-109, 2012.

25. Lan CC, Wu CS, Chiou MH, Chiang TY and Yu HS: Low-energy helium-neon laser induces melanocyte proliferation via interaction with type IV collagen: Visible light as a therapeutic option for vitiligo. Br J Dermatol 161: 273-280, 2009.

26. Goldstein NB, Koster MI, Hoaglin LG, Spoelstra NS, Kechris KJ, Robinson SE, Robinson WA, Roop DR, Norris DA and Birlea SA: Narrow band ultraviolet $B$ treatment for human vitiligo is associated with proliferation, migration, and differentiation of melanocyte precursors. J Invest Dermatol 135: 2068-2076, 2015.
27. Song Y, Zhong M and Cai FC: Oxcarbazepine causes neurocyte apoptosis and developing brain damage by triggering $\mathrm{Bax} / \mathrm{Bcl}-2$ signaling pathway mediated caspase 3 activation in neonatal rats. Eur Rev Med Pharmacol Sci 22: 250-261, 2018.

28. Hu PF, Chen WP, Bao JP and Wu LD: Paeoniflorin inhibits IL-1 $\beta$-induced chondrocyte apoptosis by regulating the Bax/ Bcl-2/caspase-3 signaling pathway. Mol Med Rep 17: 6194-6200, 2018.

29. Keswell D, Davids LM and Kidson SH: Migration of human melanocytes into keratinocyte monolayers in vitro. J Dermatol Sci 66: 160-163, 2012.

30. AlGhamdi KM, Kumar A, Ashour AE and AlGhamdi AA: A comparative study of the effects of different low-level lasers on the proliferation, viability, and migration of human melanocytes in vitro. Lasers Med Sci 30: 1541-1551, 2015.

(c) (i) (9) This work is licensed under a Creative Commons C. International (CC BY-NC-ND 4.0) License. 